PROCEEDINGS OF THE

AMERICAN MATHEMATICAL SOCIETY

Volume 139, Number 12, December 2011, Pages 4401-4407

S 0002-9939(2011)10857-8

Article electronically published on April 25, 2011

\title{
HYPERBOLIZING METRIC SPACES
}

\author{
ZAIR IBRAGIMOV \\ (Communicated by Mario Bonk) \\ Dedicated to Fred Gehring on the occasion of his 85th birthday
}

\begin{abstract}
It was proved by M. Bonk, J. Heinonen and P. Koskela that the quasihyperbolic metric hyperbolizes (in the sense of Gromov) uniform metric spaces. In this paper we introduce a new metric that hyperbolizes all locally compact noncomplete metric spaces. The metric is generic in the sense that (1) it can be defined on any metric space; (2) it preserves the quasiconformal geometry of the space; (3) it generalizes the $j$-metric, the hyperbolic cone metric and the hyperbolic metric of hyperspaces; and (4) it is quasi-isometric to the quasihyperbolic metric of uniform metric spaces. In particular, the Gromov hyperbolicity of these metrics also follows from that of our metric.
\end{abstract}

\section{INTRODUCTION}

Suppose that $(Z, d)$ is a locally compact noncomplete metric space. Let $\bar{Z}$ be its metric completion and let $\partial Z=\bar{Z} \backslash Z$. The quantities

$$
\frac{1}{\operatorname{dist}(z, \partial Z)} \quad \text { and } \quad \frac{d(x, y)}{\operatorname{dist}(x, \partial Z) \operatorname{dist}(y, \partial Z)}
$$

are ubiquitous in geometric function theory. They are used in the definitions of various metrics such as the hyperbolic cone metric (see [2]), the $j$-metric (see [3]), the quasihyperbolic metric (see [4) and the hyperbolic metric of hyperspaces (see [7]). The purpose of this paper is to show that the metric $u_{Z}$, defined by

$$
u_{Z}(x, y)=2 \log \frac{d(x, y)+\max \{\operatorname{dist}(x, \partial Z), \operatorname{dist}(y, \partial Z)\}}{\sqrt{\operatorname{dist}(x, \partial Z) \operatorname{dist}(y, \partial Z)}},
$$

hyperbolizes the space $Z$ without changing its quasiconformal geometry (see Theorem 2.1) and to show that the metrics mentioned above are quasi-isometric to $u_{Z}$ which, in particular, implies their Gromov hyperbolicity (see Section 3). The metric $u_{Z}$ and some of the results of this paper were first announced in 6 .

\section{HYPERBolizATION}

Let $(Z, d)$ be an arbitrary metric space. The distance from a point $z \in Z$ to a set $A \subset Z$ is denoted by $\operatorname{dist}(z, A)$. The diameter of a set $A \subset Z$ is denoted by

Received by the editors September 9, 2010 and, in revised form, October 20, 2010.

2010 Mathematics Subject Classification. Primary 30F45; Secondary 53C23, 30C99.

Key words and phrases. Metric spaces, Gromov hyperbolicity, quasihyperbolic metric.

(C)2011 American Mathematical Society Reverts to public domain 28 years from publication 
$\operatorname{diam}(A)$. The Hausdorff distance $d_{H}(A, B)$ between $A$ and $B$ is given by

$$
d_{H}(A, B)=\left[\sup _{a \in A} \operatorname{dist}(a, B)\right] \vee\left[\sup _{b \in B} \operatorname{dist}(b, A)\right] .
$$

Here and in what follows, we set $r \vee s=\max \{r, s\}$ and $r \wedge s=\min \{r, s\}$.

Given $x, y, z \in Z$, the quantity $(x \mid y)_{z}=[d(x, z)+d(y, z)-d(x, y)] / 2$ is called the Gromov product of $x$ and $y$ with respect to $z$. The space $Z$ is called Gromov hyperbolic if there exists $\delta \geq 0$ such that

$$
(x \mid y)_{w} \geq(x \mid z)_{w} \wedge(z \mid y)_{w}-\delta
$$

for all $x, y, z, w \in Z$. We also say that $Z$ is $\delta$-hyperbolic and refer to (2.1) as the $\delta$-hyperbolicity condition.

Let $\left(Z^{\prime}, d^{\prime}\right)$ be another metric space. A homeomorphism $f: Z \rightarrow Z^{\prime}$ is called $K$-quasiconformal, $K \geq 1$, if for each $x \in Z$ we have

$$
\limsup _{r \rightarrow 0} \frac{\sup \left\{d^{\prime}(f(x), f(y)): d(x, y) \leq r\right\}}{\inf \left\{d^{\prime}(f(x), f(y)): d(x, y) \geq r\right\}} \leq K .
$$

A map $g: Z \rightarrow Z^{\prime}$ is called quasi-isometric if there exist $k \geq 0$ and $\lambda \geq 1$ such that $\operatorname{dist}\left(x^{\prime}, g(Z)\right) \leq k$ for each $x^{\prime} \in Z^{\prime}$ and for all $x, y \in Z$ we have

$$
\frac{1}{\lambda} d(x, y)-k \leq d^{\prime}(g(x), g(y)) \leq \lambda d(x, y)+k .
$$

Let $M$ be a nonempty closed proper subset of $Z$. For convenience we put $d_{M}(z)=$ $\operatorname{dist}(z, M)$. For $x, y \in Z \backslash M$ we define

$$
u_{Z}(x, y)=2 \log \frac{d(x, y)+d_{M}(x) \vee d_{M}(y)}{\sqrt{d_{M}(x) d_{M}(y)}} .
$$

Note that $d_{M}(x)>0$ for each $x \in Z \backslash M$. Clearly, $u_{Z}(x, y) \geq 0, u_{Z}(x, y)=u_{Z}(y, x)$ and $u_{Z}(x, y)=0$ if and only if $x=y$. We have the following two lower bounds for $u_{Z}$ valid for all $x, y \in Z \backslash M$ (compare to [1, (2.3) and (2.4)]).

$$
\left|\log \frac{d_{M}(x)}{d_{M}(y)}\right|=2 \log \frac{d_{M}(x) \vee d_{M}(y)}{\sqrt{d_{M}(x) d_{M}(y)}} \leq u_{Z}(x, y)
$$

and

$$
\log \left(1+\frac{d(x, y)}{d_{M}(x)}\right) \leq \log \left(1+\frac{d(x, y)}{d_{M}(x)}\right)\left(1+\frac{d(x, y)}{d_{M}(y)}\right) \leq u_{Z}(x, y) .
$$

Observe also that the function $d_{M}: Z \rightarrow[0, \infty)$ is continuous. In fact,

$$
\left|d_{M}(x)-d_{M}(y)\right| \leq d(x, y) \quad \text { for all } \quad x, y \in Z .
$$

In particular, given $x \in Z \backslash M$, we have

$$
u_{Z}(x, y) \leq \log \frac{\left[2 d(x, y)+d_{M}(x)\right]^{2}}{d_{M}(x)\left[d_{M}(x)-d(x, y)\right]}
$$

for all $y \in Z \backslash M$ with $d(x, y)<d_{M}(x)$.

Theorem 2.1. Let $(Z, d)$ be an arbitrary metric space and let $M$ be a nonempty closed proper subset of $Z$. Then

(1) $u_{Z}$ is a metric on $Z \backslash M$;

(2) the space $\left(Z \backslash M, u_{Z}\right)$ is $\delta$-hyperbolic with $\delta \leq \log 4$;

(3) the identity map between $(Z \backslash M, d)$ and $\left(Z \backslash M, u_{Z}\right)$ is 5-quasiconformal;

(4) if the space $(Z, d)$ is complete, then so is $\left(Z \backslash M, u_{Z}\right)$. 
Proof. To prove (1), we only need to verify the triangle inequality. Given $x, y, z \in$ $Z \backslash M$, it is easy to see that

$$
\left[d_{M}(x) \vee d_{M}(z)\right] d(y, z)+\left[d_{M}(y) \vee d_{M}(z)\right] d(x, z) \geq d_{M}(z) d(x, y)
$$

and

$$
\left[d_{M}(x) \vee d_{M}(z)\right]\left[d_{M}(y) \vee d_{M}(z)\right] \geq\left[d_{M}(x) \vee d_{M}(y)\right] d_{M}(z)
$$

Hence

$\left[d(x, z)+d_{M}(x) \vee d_{M}(z)\right]\left[d(y, z)+d_{M}(y) \vee d_{M}(z)\right] \geq\left[d(x, y)+d_{M}(x) \vee d_{M}(y)\right] d_{M}(z)$, which implies $u_{Z}(x, y) \leq u_{Z}(x, z)+u_{Z}(y, z)$. Thus, $u_{Z}$ is a metric.

To prove (2), we show that $u_{Z}$ satisfies (2.1) with $\delta=\log 4$. Put $\mu(x, y)=$ $d(x, y)+d_{M}(x) \vee d_{M}(y)$ and observe that $\mu(x, y) \geq 0, \mu(x, y)=\mu(y, x)$ and that $\mu$ satisfies the triangle inequality. Let $x, y, z, w$ be arbitrary points in $Z \backslash M$. By [7, Lemma 3.7] we have $\mu(x, y) \mu(z, w) \leq 4[\mu(x, z) \mu(y, w) \vee \mu(x, w) \mu(y, z)]$. Then

$$
\frac{1}{\mu(x, y) \mu(z, w)} \geq \frac{1}{4}\left[\frac{1}{\mu(x, z) \mu(y, w)} \wedge \frac{1}{\mu(x, w) \mu(y, z)}\right]
$$

or, equivalently,

$$
\frac{\mu(x, w) \mu(y, w)}{\mu(x, y)} \geq \frac{1}{4}\left[\frac{\mu(x, w) \mu(z, w)}{\mu(x, z)} \wedge \frac{\mu(y, w) \mu(z, w)}{\mu(y, z)}\right] .
$$

Hence

$$
\begin{aligned}
(x \mid y)_{w}=\log \frac{\mu(x, w) \mu(y, w)}{\mu(x, y) d_{M}(w)} & \geq \log \frac{\mu(x, w) \mu(z, w)}{\mu(x, z) d_{M}(w)} \wedge \log \frac{\mu(y, w) \mu(z, w)}{\mu(y, z) d_{M}(w)}-\log 4 \\
& =(x \mid z)_{w} \wedge(y \mid z)_{w}-\log 4
\end{aligned}
$$

as required.

To show (3), we observe that $u_{Z}(x, y) \rightarrow 0$ if and only if $d(x, y) \rightarrow 0$, which follows from (2.4) and (2.6). Then both the identity map and its inverse are continuous. Hence the identity map is a homeomorphism. Now fix $x \in Z \backslash M$ and let $r<d_{M}(x)$. Using (2.4) we obtain

$$
\inf _{d(x, y) \geq r} u_{Z}(x, y) \geq \inf _{d(x, y) \geq r} \log \frac{d(x, y)+d_{M}(x)}{d_{M}(x)} \geq \log \frac{r+d_{M}(x)}{d_{M}(x)} .
$$

Similarly, using (2.6) we obtain

$$
\sup _{d(x, y) \leq r} u_{Z}(x, y) \leq \sup _{d(x, y) \leq r} \log \frac{\left[2 d(x, y)+d_{M}(x)\right]^{2}}{d_{M}(x)\left[d_{M}(x)-d(x, y)\right]} \leq \log \frac{\left[2 r+d_{M}(x)\right]^{2}}{d_{M}(x)\left[d_{M}(x)-r\right]} .
$$

Using, for instance, L'Hôpital's Rule one can easily show that the quotient of the second logarithmic function to the first tends to 5 as $r$ tends to 0 . Thus,

$$
\limsup _{r \rightarrow 0} \frac{\sup \left\{u_{Z}(x, y): d(x, y) \leq r\right\}}{\inf \left\{u_{Z}(x, y): d(x, y) \geq r\right\}} \leq 5 .
$$

The proof of (4) is similar to that of [1, Proposition 2.8]. Let $\left\{x_{n}\right\}$ be a Cauchy sequence in $\left(Z \backslash M, u_{Z}\right)$. It follows from (2.3) and (2.4) that

$$
0<r=\inf _{n} d_{M}\left(x_{n}\right) \leq \sup _{n} d_{M}\left(x_{n}\right)=R<\infty
$$

and that

$$
d\left(x_{n}, x_{m}\right) \leq d_{M}\left(x_{n}\right)\left(e^{u_{Z}\left(x_{n}, x_{m}\right)}-1\right) \leq R\left(e^{u_{Z}\left(x_{n}, x_{m}\right)}-1\right) .
$$


Hence $\left\{x_{n}\right\}$ is a Cauchy sequence in $(Z, d)$. Since $(Z, d)$ is complete, $\left\{x_{n}\right\}$ converges to some point $x$ in $Z$. Since $d_{M}\left(x_{n}\right) \geq r>0$, it follows from (2.5) that $d_{M}(x)>0$, i.e., $x \in Z \backslash M$. Since the identity map between $(Z \backslash M, d)$ and $\left(Z \backslash M, u_{Z}\right)$ is continuous, $\left\{x_{n}\right\}$ converges to $x$ in $\left(Z \backslash M, u_{Z}\right)$, as required.

Observe that the identity map is not, in general, conformal; i.e., given $x \in Z \backslash M$, the limit

$$
\lim _{y \rightarrow x} \frac{u_{Z}(x, y)}{d(x, y)} \quad \text { does not always exist. }
$$

Indeed, if $y$ approaches $x$ so that $d_{M}(y)=d_{M}(x)$, then

$$
\lim _{y \rightarrow x} \frac{u_{Z}(x, y)}{d(x, y)}=\lim _{y \rightarrow x} \frac{2}{d(x, y)} \log \frac{d(x, y)+d_{M}(x)}{d_{M}(x)}=\frac{2}{d_{M}(x)} ;
$$

while if $y$ approaches $x$ so that $d_{M}(y)=d_{M}(x)+d(x, y)$, then

$$
\lim _{y \rightarrow x} \frac{u_{Z}(x, y)}{d(x, y)}=\lim _{y \rightarrow x} \frac{2}{d(x, y)} \log \frac{2 d(x, y)+d_{M}(x)}{\sqrt{d_{M}(x)\left[d_{M}(x)+d(x, y)\right]}}=\frac{3}{d_{M}(x)} .
$$

\section{RELATiOns TO OtheR $\delta$-HYPERBolic METRICS}

In this section we obtain bounds for the metric $u_{Z}$ in terms of the $j$-metric, the quasihyperbolic metric, the hyperbolic cone metric and the hyperbolic metric of hyperspaces, respectively. As a consequence of these bounds we obtain alternative proofs of the $\delta$-hyperbolicity of these metrics.

For proper subdomains of Euclidean spaces, the quasihyperbolic metric and the $j$-metric were introduced by F. Gehring and B. Palka (see [4) and F. Gehring and B. Osgood (see [3), respectively. The $\delta$-hyperbolicity of the $j$-metric in such domains was proved by P. Hästö (see 5, Theorem 1]). The $\delta$-hyperbolicity of the quasihyperbolic metric in uniform domains in Euclidean spaces was proved by M. Bonk, J. Heinonen and P. Koskela (see [1, Theorem 3.6]), which was extended to uniform domains in Banach spaces by J. Väisälä (see [8, Theorem 2.12]).

3.1. The $j$-metric. Let $(Z, d)$ be an arbitrary metric space and let $M$ be a nonempty closed proper subset of $Z$. For $x, y \in Z \backslash M$ we define

$$
j_{Z}(x, y)=\frac{1}{2} \log \left(1+\frac{d(x, y)}{d_{M}(x)}\right)\left(1+\frac{d(x, y)}{d_{M}(y)}\right) .
$$

Clearly, $j_{Z}(x, y)=j_{Z}(y, x)$ and $j_{Z}(x, y)=0$ if and only if $x=y$. Since the $j$-metric has not been considered in this setting before, we will show that $j_{Z}$ is indeed a metric. That is, we verify that $j_{Z}$ satisfies the triangle inequality. Let $x, y, z \in Z \backslash M$ be arbitrary points. It follows from (2.5) that

$$
d_{M}(z)(d(x, z)+d(y, z)) \leq d_{M}(x) d(y, z)+d_{M}(z) d(x, z)+d(x, z) d(y, z) .
$$

In particular,

$$
d_{M}(z)\left(d_{M}(x)+d(x, y)\right) \leq\left(d_{M}(x)+d(x, z)\right)\left(d_{M}(z)+d(y, z)\right) .
$$

Dividing both sides by $d_{M}(x) d_{M}(z)$ we obtain

$$
\left(1+\frac{d(x, y)}{d_{M}(x)}\right) \leq\left(1+\frac{d(x, z)}{d_{M}(x)}\right)\left(1+\frac{d(y, z)}{d_{M}(z)}\right)
$$


Similarly, we obtain

$$
\left(1+\frac{d(x, y)}{d_{M}(y)}\right) \leq\left(1+\frac{d(x, z)}{d_{M}(z)}\right)\left(1+\frac{d(y, z)}{d_{M}(y)}\right) .
$$

Combining the last two inequalities we obtain $j_{Z}(x, y) \leq j_{Z}(x, z)+j_{Z}(z, y)$.

Theorem 3.1. For all $x, y \in Z \backslash M$ we have

$$
2 j_{Z}(x, y) \leq u_{Z}(x, y) \leq 2 j_{Z}(x, y)+2 \log 2 .
$$

In particular, the space $\left(Z \backslash M, j_{Z}\right)$ is $\delta$-hyperbolic with $\delta \leq \frac{5}{2} \log 2$.

Proof. It follows from (2.5) that $d_{M}(x) \vee d_{M}(y) \leq d_{M}(x) \wedge d_{M}(y)+d(x, y)$ for all $x, y \in Z \backslash M$. Hence

$$
\left[d(x, y)+d_{M}(x) \vee d_{M}(y)\right]^{2} \leq 4\left[d_{M}(x)+d(x, y)\right]\left[d_{M}(y)+d(x, y)\right] .
$$

In particular,

$$
u_{Z}(x, y) \leq \log \left(1+\frac{d(x, y)}{d_{M}(x)}\right)\left(1+\frac{d(x, y)}{d_{M}(y)}\right)+2 \log 2 .
$$

Combining (2.4) and (3.3), we obtain (3.2). The latter in combination with Theorem 2.1 implies the second part.

3.2. The quasihyperbolic metric. Let $(Z, d)$ be a locally compact rectifiably connected noncomplete metric space and let $\partial Z=\bar{Z} \backslash Z$, where $\bar{Z}$ is the metric completion of $Z$. The quasihyperbolic metric is defined by

$$
k_{Z}(x, y)=\inf _{\gamma} \int_{\gamma} \frac{|d z|}{\operatorname{dist}(z, \partial Z)},
$$

where the infimum is taken over all rectifiable curves $\gamma$ joining the points $x$ and $y$ in $Z$. In this setting the quasihyperbolic metric was studied by M. Bonk, J. Heinonen and P. Koskela (see [1]). Recall that the space $Z$ is called $A$-uniform $(A \geq 1)$ if every pair of points in $Z$ can be joined by a curve $\gamma:[0,1] \rightarrow Z$ such that $\operatorname{length}(\gamma) \leq A d(\gamma(0), \gamma(1))$ and

$$
\operatorname{length}(\gamma \mid[0, t]) \wedge \operatorname{length}(\gamma \mid[t, 1]) \leq A \operatorname{dist}(\gamma(t), \partial Z)
$$

for all $t \in[0,1]$ (see [1, Definition 1.9]). It follows from [1, (2.4) and (2.16)] that

$$
j_{Z}(x, y) \leq k_{Z}(x, y) \leq 4 A^{2} j_{Z}(x, y)
$$

for all $x, y \in Z$. Note that the second inequality holds under the assumption that $Z$ is $A$-uniform. In particular, the space $\left(Z, k_{Z}\right)$ is proper, geodesic and $\delta$-hyperbolic provided $Z$ is uniform (see [1, Theorem 3.6]).

Now if $Z$ is $A$-uniform, then it follows from (3.2) and (3.4) that

$$
\frac{1}{2 A^{2}} k_{Z}(x, y) \leq u_{Z}(x, y) \leq 2 k_{Z}(x, y)+2 \log 2
$$

for all $x, y \in Z$. (Here the metric $u_{Z}$ is as in (1.1).) Hence both the identity map $\operatorname{id}_{Z}:\left(Z, k_{Z}\right) \rightarrow\left(Z, u_{Z}\right)$ and its inverse are quasi-isometric. In particular, as $\left(Z, k_{Z}\right)$ is geodesic and quasi-isometric to $\left(Z, u_{Z}\right)$, an argument due to M. Bonk gives an alternative proof of the $\delta$-hyperbolicity of $k_{Z}$ (see [5, Lemma 4]). 
3.3. The hyperbolic cone metric. Let $(X, d)$ be a bounded metric space and let $\operatorname{Con}(X)=X \times(0, \operatorname{diam}(X)]$. The hyperbolic cone metric is defined by

$$
\rho((x, r),(y, s))=2 \log \frac{d(x, y)+r \vee s}{\sqrt{r s}} .
$$

The space $(\operatorname{Con}(X), \rho)$ is $\delta$-hyperbolic for some $\delta$ (see [2, Theorem 7.2]). Our computations imply that $\delta \leq 5 \log 2$ (see Theorem 3.2).

We consider the space $Z=X \times[0, \operatorname{diam}(X)]$ equipped with the metric $d^{\prime}$,

$$
d^{\prime}((x, r),(y, s))=d(x, y)+|r-s|,
$$

and let $M=X \times\{0\}$ so that $\operatorname{Con}(X)=Z \backslash M$. Observe that for each $(x, r) \in$ $\operatorname{Con}(X)$, we have $\inf \left\{d^{\prime}((x, r),(y, 0)):(y, 0) \in M\right\}=\inf _{y \in X}[d(x, y)+r]=r$. Applied to the space $\left(Z, d^{\prime}\right)$ and the subset $M \subset Z$, the metric $u_{Z}$ takes the form

$$
u_{Z}((x, r),(y, s))=2 \log \frac{d(x, y)+|r-s|+r \vee s}{\sqrt{r s}} .
$$

Since $|r-s| \leq r \vee s$, we obtain the following result.

Theorem 3.2. For all $x, y \in Z \backslash M$ we have

$$
\rho(x, y) \leq u_{Z}(x, y) \leq \rho(x, y)+2 \log 2 .
$$

In particular, the space $(\operatorname{Con}(X), \rho)$ is $\delta$-hyperbolic with $\delta \leq 5 \log 2$.

3.4. The hyperbolic metric of hyperspaces. We recall the hyperbolization of hyperspaces from [7]. Let $(X, d)$ be an arbitrary metric space and let $\mathcal{H}(X)$ be the hyperspace of all nondegenerate closed bounded subsets of $X$ equipped with the metric $d_{\mathcal{H}}$,

$$
d_{\mathcal{H}}(A, B)=2 \log \frac{d_{H}(A, B)+\operatorname{diam}(A) \vee \operatorname{diam}(B)}{\sqrt{\operatorname{diam}(A) \operatorname{diam}(B)}} .
$$

The space $\left(\mathcal{H}(X), d_{\mathcal{H}}\right)$ is $\delta$-hyperbolic with $\delta \leq 2 \log 2$ (see [7, Theorem 4.7]).

Now let $Z$ be the set of all nonempty closed bounded subsets of $X$ endowed with the Hausdorff metric $d_{H}$. Let $M=Z \backslash \mathcal{H}(X)$ so that $\mathcal{H}(X)=Z \backslash M$. Observe that for any $A \in \mathcal{H}(X)$ we have $d_{M}(A)=\inf \left\{d_{H}(A,\{x\}): x \in X\right\}$ and $\operatorname{diam}(A) / 2 \leq d_{M}(A) \leq \operatorname{diam}(A)$. Applied to the space $\left(Z, d_{H}\right)$ and the subset $M \subset Z$, the metric $u_{Z}$ takes the form

$$
u_{Z}(A, B)=2 \log \frac{d_{H}(A, B)+d_{M}(A) \vee d_{M}(B)}{\sqrt{d_{M}(A) d_{M}(B)}} .
$$

Consequently, we obtain the following result.

Theorem 3.3. For all $A, B \in \mathcal{H}(X)$ we have

$$
d_{\mathcal{H}}(A, B)-2 \log 2 \leq u_{Z}(A, B) \leq d_{\mathcal{H}}(A, B)+2 \log 2 .
$$

\section{REFERENCES}

[1] M. Bonk, J. Heinonen and P. Koskela, Uniformizing Gromov hyperbolic spaces, Astérisque (2001), no. 270, viii+99. MR1829896 (2003b:30024)

[2] M. Bonk and O. Schramm, Embeddings of Gromov hyperbolic spaces, GAFA, Geom. Func. Anal., vol. 10 (2000), 266-306. MR1771428 (2001g:53077)

[3] F. W. Gehring and B. G. Osgood, Uniform domains and the quasihyperbolic metric, J. Analyse Math., vol. 36 (1979), 50-74. MR.581801 (81k:30023)

[4] F. W. Gehring and B. P. Palka, Quasiconformally homogeneous domains, J. Analyse Math., vol. 30 (1976), 172-199. MR0437753 (55:10676) 
[5] P. Hästö, Gromov hyperbolicity of the $j_{G}$ and $\tilde{j}_{G}$ metrics, Proc. Amer. Math. Soc., vol. 134 (2006), 1137-1142. MR2196049 (2007g:30062)

[6] Z. Ibragimov, A canonical $\delta$-hyperbolic metric for metric spaces, Abstracts, International Congress of Mathematicians, Hyderabad, India (2010), 209-210.

[7] Z. Ibragimov, Hyperbolizing hyperspaces, Michigan Math. J., vol. 60, no. 1 (2011) (to appear).

[8] J. Väisälä, Hyperbolic and uniform domains in Banach spaces, Ann. Acad. Sci. Fenn. Math., vol. 30 (2005), 261-302. MR2173365 (2006k:30025)

Department of Mathematics, California State University, Fullerton, California 92831

E-mail address: zibragimov@fullerton.edu 